\title{
Brain Disorders and its Treatment
}

\section{Manu Mitra*}

Department of Alumnus with Electrical Engineering, University of Bridgeport, USA

*Corresponding author: Manu Mitra, Department of Alumnus with Electrical Engineering, University of Bridgeport, USA.
Received Date: April 09, 2019

Published Date: April 17, 2019

\section{Introduction}
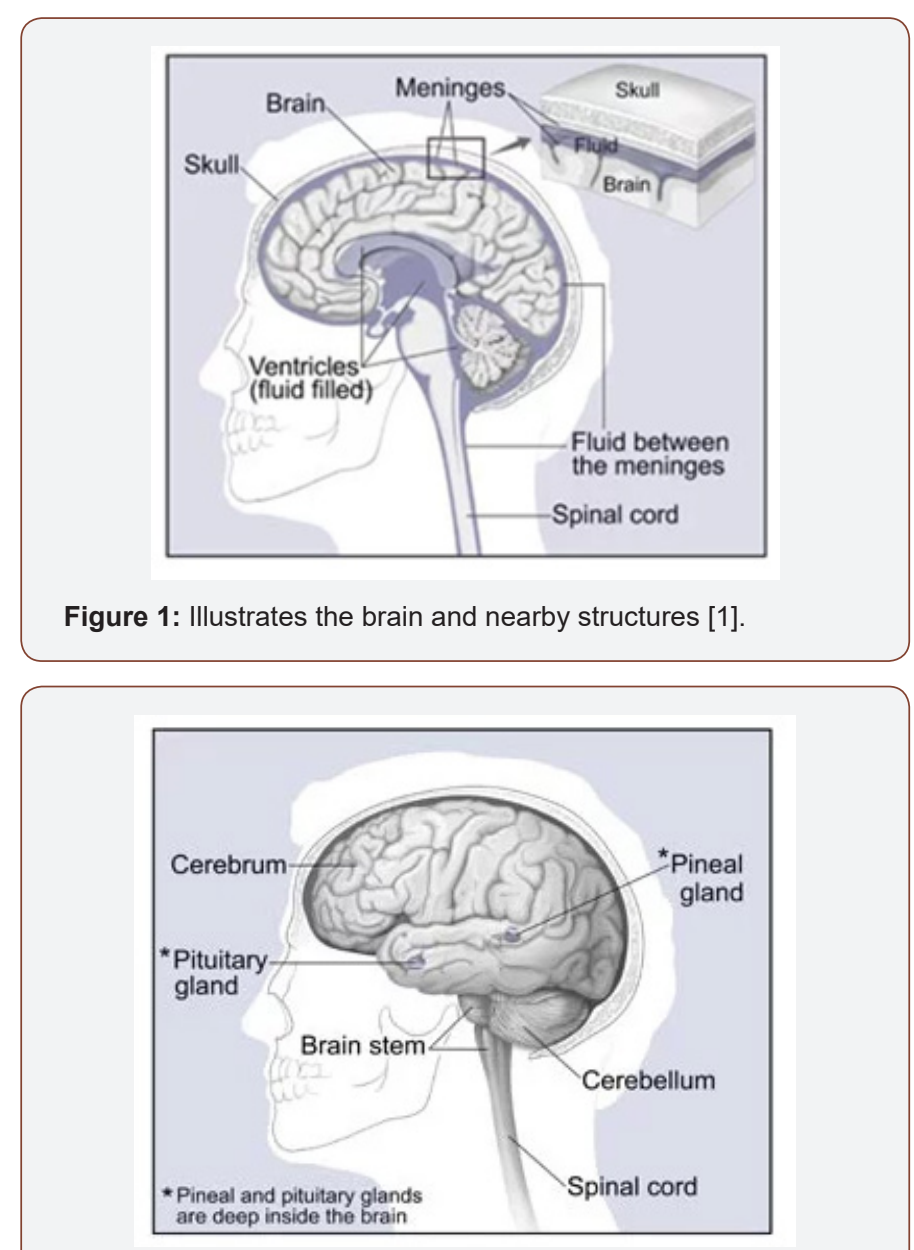

Figure 2: Illustrates the major parts of the brain [1].

LBrain is the collection of supportive tissues and nerve cells like meninges and glial cells [1]. Diagnostic test is to take a brain scan that takes pictures of the brain; similar to X-ray that can see inside the body to diagnose a broken bone, a brain scan can see inside the skull. Two major scans for diagnosing a brain are Computed Tomography (CT) and Magnetic Resonance Imaging (MRI). Brain scans are normally painless and non-invasive. The most difficult part is to sit or lie for a long time and some patients may also need medicines for claustrophobia. Computer Tomography (CT) scan integrates high speed X-ray technology with a computer. CT scanner circles the head, taking scanning every angle. Magnetic Resonance Imaging (MRI) uses magnets and radio waves to generate pictures of the brain tissues. MRI scans create two dimensional or three dimensional pictures of the brain and unlike CT scans, it does not use radiations [2] (Figure 1\&2).

\section{Cure for Brain Diseases is One Step Closer?}

Numerous nerve cells make up the brain control; the role of the brain through synapses. Studies demonstrate that synaptic binding proteins plays a significant role in formation of synapses. But detail information about the processes for collectively controlling the synapses remain unknown. Research team led by Prof. Jaewon Ko and Ji Won Um from department of brain and cognitive sciences analyzed a new method of formation of brain synapses during synaptic binding protein complexes. Research team has been concentrating on related protein and discovering detailed mechanisms to identify principles of formation of excitatory synapses in between another synapses. In this research, they found that the interaction between PTP $\sigma$ proteins and some bone proteins and it plays a significant role in synapse formation. To be certain, they were able to analyze that the 'normal tyrosine signaling mechanism' resulting from the reactions of certain elements of PTP $\sigma$ proteins is most vital component of synapse composition.

As the recent study has stated, РTP $\sigma$ proteins along with neurexin, are treated as key proteins responsible for the development of neural circuits. Their research team will continue further studies on development of synapses and neural circuits was stated by Prof. Ko [3,4] (Figure 3). 

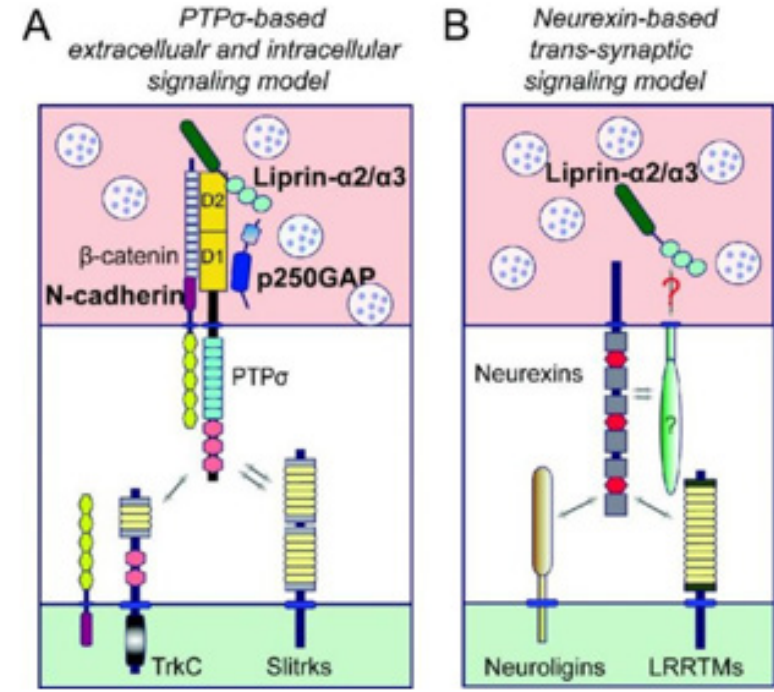

Figure 3: Illustrates Molecular model of PTP signaling pathways in heterologous synapse formation. A) PTP triggers excitatory heterologous synapse formation through a combination of extracellular and intracellular signaling components. The PTP D2 domain binds intracellular adaptor proteins and substrates to recruit the vesicular machinery for excitatory synapse development. This signal transduction model differs from that of neurexin. B) Neurexins serve as anchor proteins that transduce postsynaptic signals from various ligands (e.g., neuroligins and leucine-rich repeat transmembrane proteins) and transfer them to adjacent, but as yet unidentified, coreceptor proteins to mediate the signal transduction cascades necessary for full heterologous synapse formation activity. Image Credit: Daegu Gyeongbuk Institute of Science and Technology (DGIST) [4].

Treatment for Lesser Known Cells Crucial for Brain Development

In the research, a student from Stanford's, Steven Sloan, led a series of investigations differentiating astrocytes from hCSs to those discovered in tissue from progressing and adult human brain. The research team grew hCSs for approximately 20 months, one of the longest ever lab grown human brain cells. The conclusion confirmed that the lab grown cells change over time in an identical manner to the cells taken directly from brain tissue during very initial life, a critical time for brain development. This method is considered critical for normal brain growth and developments are thought to cause range of mental health and neurological disorders, like autism and schizophrenia. Building hCSs utilizing cells from patients could permit researchers to uncover the basic development biology at the core of these disorders. The research demonstrates that hCS-grown astrocytes advance at the same rate as those found in human brains, in terms of their shapes, gene activity and their functions. For instance, astrocytes taken from hCSs that were less than 6 months old multiplied briskly and were highly busy in eradicating unnecessary contacts between neurons. Astrocytes expanded in hCSs for more than 9 months could not portray and removed significantly fewer of those contacts, imitating astrocytes in infants 6 to 12 months old. However, astrocytes from expanding and adult brains, the early and late stage astrocytes from hCSs were equally competent at encouraging new contact to form between neurons. Astrocytes are not just bystanders in the brain, they participate actively in neurological function was confirmed by Dr. Pasca [5,6] (Figure 4).

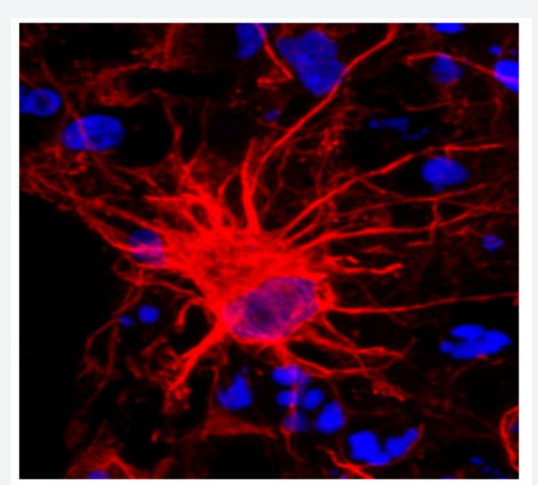

Figure 4: Illustrates NIH-funded researchers used 3-D collections of brain tissue grown from human cells to study the brain's starshaped astrocytes. Image Credit: courtesy of Sergiu Pasca, M.D., Stanford University [6].

\section{Mystic Mossy Cells for Treatment of Brain Disease}

Song and their team members used advanced tracing techniques on several different strains of genetically engineered lab mice; they described mossy cells in mouse hippocampi and marked connections to other brain cells. They discovered that mossy cells related to neural stem cells directly and indirectly. These mossy cells directly exchange information with stem cells in hippocampus via an excitatory pathway. These mossy cells also obliquely mutually exchange information with stem cells through neighboring neurons called interneurons that then associate to stem cells via inhibitory pathway. Song and her team found that mossy cells can switch the balance of their direct and indirect signaling, hence dynamically controlling neural stem cell action. In their research, Song's team discovered that moderate mossy cell activity, for instance, appeared in dominance of indirect signaling and preserved stem cells in "quiescent" state may transcend to a low level of neurogenesis, but the steam cells could be kept over the long time. However, high mossy cell activity, which could occur when the brain is stimulated or in an unhealthy state, made direct signaling dominant and preserved stem cells in an "activated" state that may transcend to a high level of neurogenesis. This may risk losing stem cells over the long time.

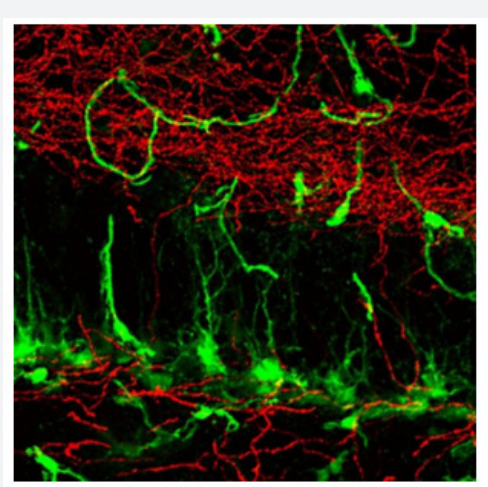

Figure 5: Illustrates the mossy cell commissural projections (red) and neural stem cells (in green) in the adult mouse dentate gyrus region of the brain. Image Credit: Song Lab, UNC School of Medicine [7]. 
They were surprised that small population of mossy cells could make such a big impact on stem cell behavior. They are beginning to look at how cell populace is changed. It was said by Song [7] (Figure $5)$.

\section{Self-Tuning Brain Implant May Help with Parkinson's Disease}

Deep brain stimulation is a technique of managing Parkinson's disease syndrome by surgically implanting an electrode, a very thin wire into the brain. Classical deep brain stimulations transfer constant stimulation to a part of the brain known as basal ganglia to treat symptoms of Parkinson's. On the other hand, this approach can transcend to unnecessary after effects, requiring reprogramming by a qualified clinician. This new technique described in this research is adaptive, so that stimulations conveyed is responsive in real time to signal received from the patient's brain. Many patients with Parkinson's disease who would have advantage from deep brain stimulation are hard to treat because too much stimulation can cause dyskinesia. Hence correct estimation level of stimulation is like trying to hit a constantly moving target. A flexible system could offer, that one being tested can be an alternative and may also limit side effects of classical deep brain stimulations, however testing is to be done. They have demonstrated the feasibility of adaptive deep brain stimulation and they are planning larger longer term trails to decide how effective this method of managing the symptoms of patients with Parkinson's disease was concluded by Dr. Starr [8,9] (Figure 6).

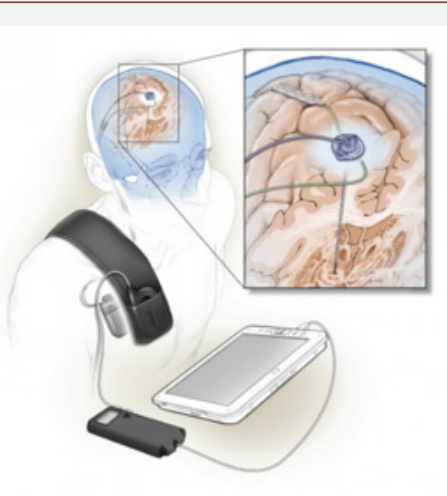

Figure 6: Illustrates Stimulating and sensing electrodes are implanted in the brain and connect to small computer under the skin. Data from this computer can be read by an external device. Image Credit: courtesy of Ken Probst/Starr lab [9].

\section{More Lives May be Saved by Brain Scan Emergency Stroke Treatment}

Ischemic stroke takes place when a cerebral blood vessel gets blocked, cutting off the transfer of oxygen and nutrients to brain tissue. Brain tissue in the existing area of the obstruction, known as the core, cannot typically be saved from dying, and it can enlarge over time. On the other hand, it has long been thought that the area neighboring the core (known as the ischemic penumbra) has the possibility to be saved based on how fast blood flow can be replaced. Over the past 20 years, researchers have been working to advance brain scanning methods called perfusion imaging that can identify patients with brain tissue that can be restored by removing the obstruction. In perfusion imaging, a standard dye is injected and scanned for a few minutes as it goes through the brain. Using an automated software called as RAPID to analyze perfusion MRI or CT scans, the DEFUSE 3 scientist recognized patients thought to have a restorable tissue up to 16 hours after stroke onset. The contenders were randomized to either accept endovascular thrombectomy plus standard medical therapy or only medical therapy. DEFUSE 3 builds on results from the various studies performed as well as industry sponsored, which used perfusion imaging technology to determine patients results most likely to aid from interventions like thrombectomy. These studies implied that the advanced brain imaging could determine which patients could assist from restoring blood flow in a continued treatment window [10,11].

\section{Acknowledgment}

Author would like to thank Prof. Navarun Gupta, Prof. Hassan Bajwa, Prof. Linfeng Zhang and Prof. Hmurcik for their academic support. Author also thanks anonymous reviewers for their comments.

\section{Conflict of Interest}

There is no conflict of interest as per Author's point of view.

\section{References}

1. Davis CP (2017) Brain Tumor: Symptoms, Signs, Treatment, Surgery \& Types. Retrieved from https://www.medicinenet.com/brain_tumor/ article.htm\#brain_tumor_primary_definition_and_facts/

2. American brain Tumor Association (2018) Brain Scans. Retrieved from https://www.abta.org/about-brain-tumors/brain-tumor-diagnosis/

3. Kyung Ah Han, Ji Seung Ko, Gopal Pramanik, Jin Young Kim, Katsuhiko Tabuchi, Ji Won Um, et al. (2018) PTPo drives excitatory presynaptic assembly via various extracellular and intracellular mechanisms. J Neurosci 38(30): 6700-6721.

4. DGIST (Daegu Gyeongbuk Institute of Science and Technology) (2018) One step closer to finding a cure for brain diseases. ScienceDaily Retrieved from www.sciencedaily.com/releases/2018/07/180713093539.htm

5. Steven A Sloan, Spyros Darmanis, Nina Huber, Themasap A Khan, Fikri Birey, et al. (2017) Human Astrocyte Maturation Captured in 3D Cerebral Cortical Spheroids Derived from Pluripotent Stem Cells. Neuron 95(4): 779-790.

6. NIH/National Institute of Neurological Disorders and Stroke (2017) Scientists give star treatment to lesser-known cells crucial for brain development: Study lays foundation for future research into the roots of several brain disorders. ScienceDaily Retrieved from www.sciencedaily. com/releases/2017/08/170816134650.htm

7. University of North Carolina Health Care (2018) Can scientists leverage mysterious mossy cells for brain disease treatments? ScienceDaily Retrieved from www.sciencedaily.com/ releases/2018/07/180726162702.htm

8. Nicole C Swann, Coralie de Hemptinne, Margaret C Thompson, Svjetlana Miocinovic, Andrew M Miller, et al. (2018) Adaptive deep brain stimulation for Parkinson's disease using motor cortex sensing. J Neural Eng 15(4): 046006.

9. NIH/National Institute of Neurological Disorders and Stroke (2018) Selftuning brain implant could help treat patients with Parkinson's disease: Key firststep to improving deep brain stimulation. ScienceDaily Retrieved from www.sciencedaily.com/releases/2018/05/180529185351.htm

10. Gregory W Albers, Michael P Marks, Stephanie Kemp, Soren Christensen, Jenny P Tsai, et al. (2018) Thrombectomy for Stroke at 6 to 16 Hours with Selection by Perfusion Imaging. N Engl J Med 378: 708-718. 
11. NIH/National Institute of Neurological Disorders and Stroke (2018) Brain-scan guided emergency stroke treatment can save more lives: Study finds patients treated up to 16 hours after stroke showed positive outcomes. ScienceDaily Retrieved from www.sciencedaily.com/ releases/2018/01/180124143704.htm 